\title{
A New Framework for Mapping Business Domain Ontologies
}

\author{
Sahar Zangenehpour \\ Department of Computer \\ Engineering \\ Science and Research Branch, \\ Islamic Azad University \\ Tehran, Iran
}

\author{
Mir Ali Seyyedi \\ Department of Computer \\ Engineering \\ Tehran Jonob Branch, Islamic \\ Azad University \\ Tehran, Iran
}

\author{
Mehran Mohsenzadeh \\ Department of Computer \\ Engineering \\ Science and Research Branch, \\ Islamic Azad University \\ Tehran, Iran
}

\begin{abstract}
In cross enterprise collaboration, business domains need to interoperate and collaborate to each other for receiving profits. To create an understanding of enterprises and the ways they do business, a starting point could be business models. The goal of business modeling is to create semantically representations of business domain concepts like transactions, processes, value chains. Business domain ontologies can be used as a reference model for concrete business models. Business domain ontology describes concepts, concept relations and axioms that are potentially relevant for business models. The three main business modeling ontologies are REA, the $\mathrm{e}^{3}$-value ontology and the Business Model Ontology (BMO).

In this paper, a mapping is proposed between these three well known business domain ontologies (REA, $\mathrm{e}^{3}$-value and BMO). The proposed mapping helps business domains with different ontologies in a cross enterprise collaboration, to be understandable and interoperable, but it does not force each business domain to have a common vocabulary and a unique ontology in order to be interoperable to each other.
\end{abstract}

\section{General Terms}

Business domain ontology mapping.

\section{Keywords}

Cross enterprise collaboration; Ontology; Interoperability; Business Modeling; Business Domain; Business Domain Ontologies; Mapping Ontology; Evidence Node

\section{INTRODUCTION}

Enterprises are interoperable in business domains and they do activities for receiving profits. Business modeling is used for defining and understanding business domains and they show business transactions and activities. Business domain ontologies, define concepts and their relations in business domains so they are used as a technique for business domain modeling. There are a number of business domain ontologies, among them the three well established ontologies are:

- $\quad$ Business Model Ontology (BMO)

- $\mathrm{e}^{3}$-value Ontology

- $\quad$ Resource Events Agents (REA) Ontology

Gordijin proposed $\mathrm{e}^{3}$-value ontology in [1]. On the one hand the $\mathrm{e}^{3}$-value ontology aims at providing a simple valueoriented approach to enhance the ways of doing business and capturing business decisions, for example who is doing what and who is offering what to whom [2]. On the other hand it facilitates profitability analysis of the created business models [2]. Osterwalder proposed Business Model Ontology (BMO) in [3]. It identifies various business concepts classified around four pillars: Product; Customer Interface; Infrastructure Management; and Financial Aspects. Altogether, these pillars aim at defining a company's business, their customers, how they carry out delivering their value proposition, who are their business partners and how they generate revenue [2]. McCarthy proposed The Resource Events Agents (REA) ontology in [4]. It centers on the concept of economic reciprocity, meaning that every economic event that increments a business's resources is linked with a decrement economic event [2]. Each ontology has its own usage. Among them the BMO is wider in scope of relationship with customers. The REA is focused on increment and decrement of an actor's resources. The $\mathrm{e}^{3}$-value aims at modeling value webs of cooperating trading partners and also helps the profitability analysis of the modeled business scenarios [2].In cross enterprise collaboration, different business domains must have interoperability to receive profits. As any business domain is based on a special ontology so various business domains could not be interoperable. Using a unique ontology is an approach for interoperability between different ontologies, although has some problems such as it is not flexible and it forces any business domain to use a unique ontology. There is also another approach which is mapping between ontologies and is flexible. There are some proposed mappings between these ontologies but none of them is a reliable mapping because all of them are based on description of the concepts in ontologies [5, 6]. For making a reliable mapping between ontologies could use a standard framework for mapping between ontologies. There are some frameworks in semantic web for mapping ontologies [7-19].

Among these frameworks, Omen is selected for two reasons:

- It is a schema based framework

- It includes association relationship

Because schema based frameworks are more general and for mapping concepts between these mentioned ontologies, association relationship is essential and it causes difference between ontology and thesaurus. For having a much correct 


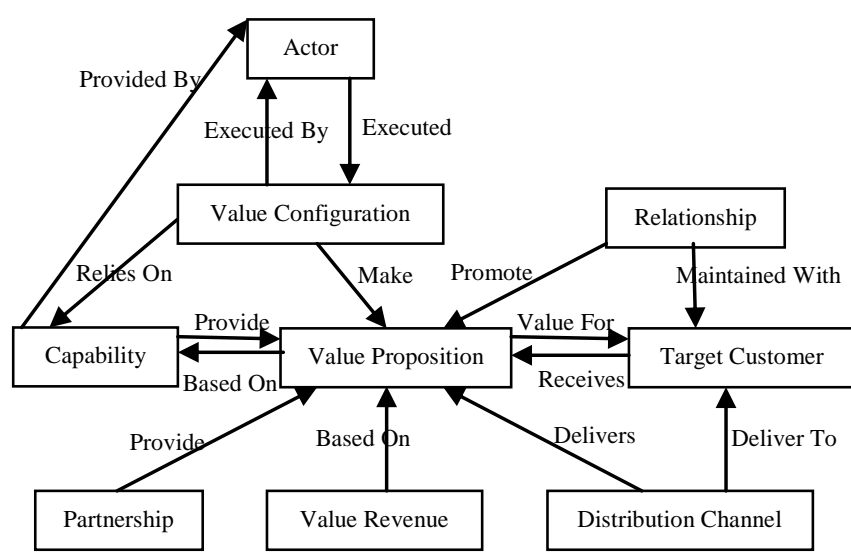

Fig 1: Concepts and their relations in BMO

Mapping between these mentioned ontologies, a Meta rule has added to omen and a new framework which is named extended omen is proposed. The paper is structured as follows. In Section 2 a description of mete rules in extended Omen. In Section 3 the usage of extended Omen for mapping between the concepts of the mentioned ontologies. In Section 4 there is the evaluation. In Section 5 there is a case study. Section 6 concludes the paper with a summary and directions for future work.

\section{Extended Omen}

Extended omen exactly like omen uses Meta rules for distribution mapping between concepts of ontologies. There are two Meta rules that are used in the proposing mapping. Omen also has the first Meta rule in extended omen, the second Meta rule is just used for extended omen and it is used for mapping between REA, BMO and $\mathrm{e}^{3}$-value ontologies. Meta rules are defined in below:

- If two concepts $\mathrm{C} 1$ and $\mathrm{C}^{\prime} 1$ match, and there is a relationship $\mathrm{q}$ between $\mathrm{C} 1$ and $\mathrm{C} 2$ in $\mathrm{O}$ and a matching relationship q' between $\mathrm{C}^{\prime} 1$ and $\mathrm{C}^{\prime} 2$ in $\mathrm{O}^{\prime}$, then we can increase the probability of match between $\mathrm{C} 2$ and $\mathrm{C}$ '2. Informally, if two nodes in ontology graph match and so do two arrows coming out of these nodes, then the probability that node at the other end of the arrows match is increased [9].

- If two concepts $\mathrm{C} 1$ and $\mathrm{C}^{\prime} 1$ match, and there is a relationship $\mathrm{q}$ between $\mathrm{C} 1$ and $\mathrm{C} 2$ in $\mathrm{O}$ and a relationship q' between $\mathrm{C}^{\prime} 1$ and $\mathrm{C}^{\prime} 2$ in $\mathrm{O}^{\prime}$, that $\mathrm{q}$ is a subset of q' then we can say $\mathrm{C} 2$ is a subclass of $\mathrm{C}^{\prime} 2$.

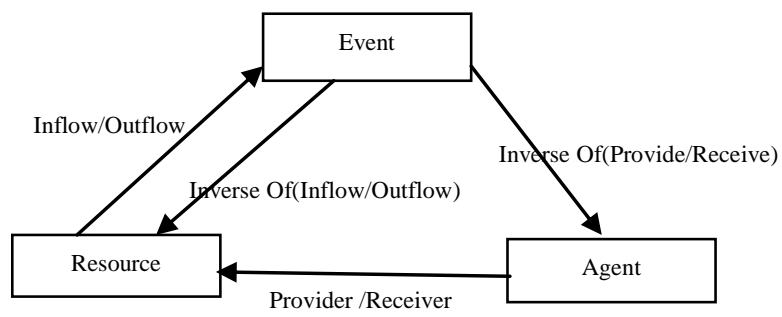

Fig 2: Concepts and their relations in REA

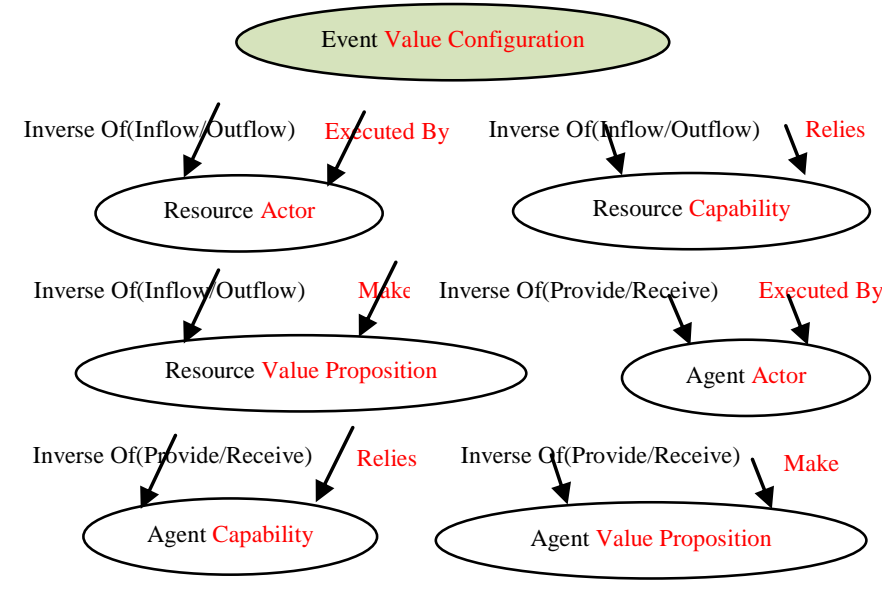

Fig 3: Nodes and their relations mapping Between REA and BMO

\section{Usage of Extended Omen for Mapping}

In this section, extended omen is used for mapping between concepts of three well established business modeling ontologies, the REA, BMO and $\mathrm{e}^{3}$-value.

\subsection{Mapping Between REA and BMO}

Based on description of concepts in [2], Fig. 1 is reached. Based on OWL specification of an example REA-structured Enterprise Schema in [20], Fig. 2 is reached. As in BMO, Value Configuration means, all activities that is executed by a firm or a partner, with the limitation of events in REA to events which are executed by a firm or a partner so Event in REA is mapped to Value Configuration in BMO. In extended Omen, the evidence node is a node with Event and Value Configuration so Fig. 3 is reached. In REA, the Inverse of Provide/Receive relationship which is from Event to Agent means, which Agent is the provider or the receiver of that event. In BMO, the Executed by relationship which is from Value Configuration to Actor means, a set of activities, are executed by which actors. As executed by in some places means provided by and in some other places means received by so according to the second Meta rule in extended omen, Executed by in BMO is a subset of Inverse of Provide/Receive in REA so Actor in BMO is a subclass of

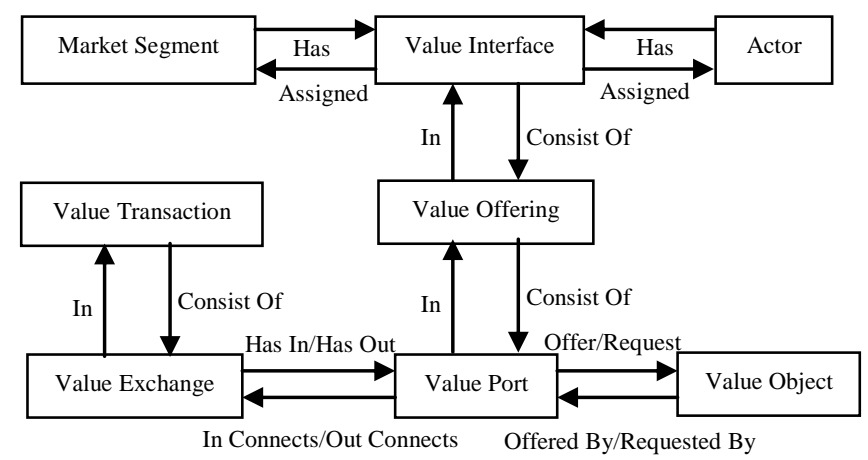

Fig 4: Concepts and their relations in $\mathrm{e}^{3}$-value in global view 


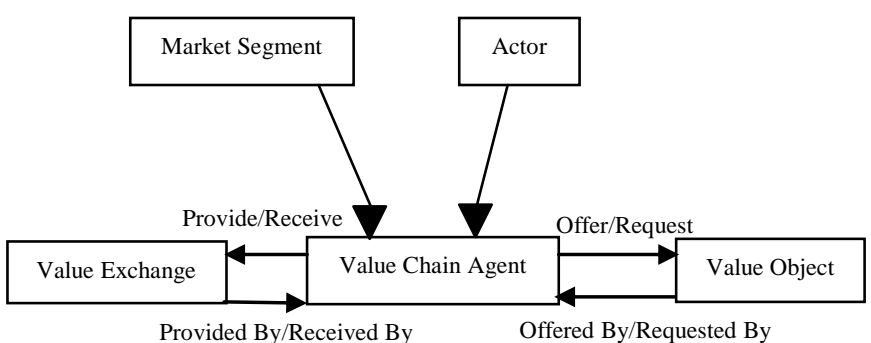

Fig 5: Concepts and their relations in $\mathrm{e}^{3}$-value with Value Chain Agent in global view

Agent in REA. In REA, the Inverse of Inflow/Outflow relationship is from Event to Resource that means with those events which resources are decreased or increased. In BMO, the Make relationship which is from Value Configuration to Value Proposition means those activities makes which Values. As every Value Proposition which is made would be offered and decreased according to the second Meta rule in extended omen, Make in BMO is a subset of Inverse of Inflow/Outflow in REA so Value Proposition in BMO is a subclass of Resource in REA.

\subsection{Mapping between REA and $\mathrm{e}^{3}$-Value}

Fig. 4 is showing concepts and their relations in $\mathrm{e}^{3}$-value in global view. Because Actor and Market Segment have equal relationships to value Interface so can define a new concept which is named Value Chain Agent and define them as subclasses of Value Chain Agent. Value Chain agent includes Value Interfaces, Value Offerings and Value Ports so can omit these concepts and put them in Value Chain Agent so fig. 5 is reached. Concepts in REA and $\mathrm{e}^{3}$-value are mapped to each other in two steps. If just consider transfer events in REA, so could map Event in REA to Value Exchange in $\mathrm{e}^{3}$-value. In the first step, the evidence node includes Event in REA and Value Exchange in $\mathrm{e}^{3}$-value and fig.6 is reached. The relationship Inverse of Provide / Receive in REA is mapped to Provided by / Received by in $\mathrm{e}^{3}$-value so can map Agent in REA and Value Chain Agent in $\mathrm{e}^{3}$-value. In the second step, one of the evidence nodes includes Event in REA and Value Exchange in $\mathrm{e}^{3}$-value, the other evidence node includes Agent in REA and Value Chain Agent in $\mathrm{e}^{3}$-value. As cannot reach more maps with the evidence node

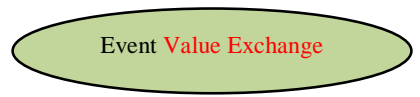

Provided By/Received By

Provided By/Received By

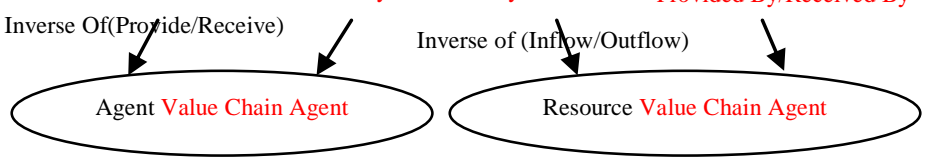

Fig 6: Nodes and their relations in Mapping between REA and $\mathrm{e}^{3}$-value in the first step

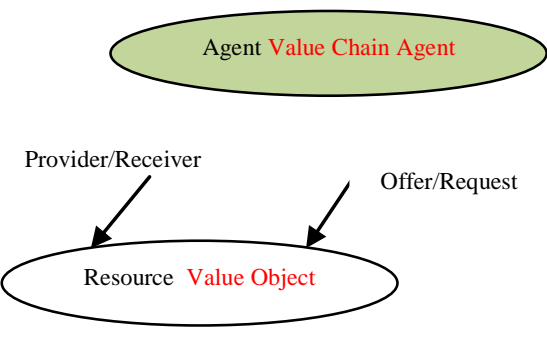

Fig 7: Nodes and their relations in Mapping Between REA and $\mathrm{e}^{3}$-value in the second step

which has Value Exchange in $\mathrm{e}^{3}$-valueand Event in REA so just consider the evidence node with Value Chain Agent in $\mathrm{e}^{3}$ value and Agent in REA and define its relationships with other nodes so fig. 7 is reached. As the limitation of events in REA to transfer events, the relationship Provider/Receiver that is from Agent to Resource means, which resources are provided or received by that agent. In transfer events, provider and receiver are equal to offer or request so can map the Provider / Receiver relation to Offer / Request and Resource in REA is mapped to Value Object in $\mathrm{e}^{3}$-value.

\subsection{Mapping between BMO and $\mathrm{e}^{3}$-Value}

Fig. 8 is showing concepts and their relations in $\mathrm{e}^{3}$-value in global and activity view. Provided by / Received by relationship that is from Value Exchange to Value Chain Agent means Value Exchange is executed by which Value Chain Agents so can replace Provided by / Received by relationship with Executed by. As Value Activity and Value Exchange have equal relations with Value Chain Agent so can define a new concept which is named Value Configuration and define them as subclasses of the new concept so fig. 9 is reached. Concepts in BMO and $\mathrm{e}^{3}$-value are mapped to each other in one step. As Actor in BMO is a firm or its partners, with the limitation of Value Chain Agent in $\mathrm{e}^{3}$-value to the firm and its partners, can map these two concepts. In the first step, the evidence node has Agent in BMO and Value Chain Agent in $\mathrm{e}^{3}$-value so fig. 10 is reached. With mapping Execute relation in $\mathrm{BMO}$ to Execute Relation in $\mathrm{e}^{3}$-value can map Value Configuration in BMO to Value Configuration in $\mathrm{e}^{3}$ value.

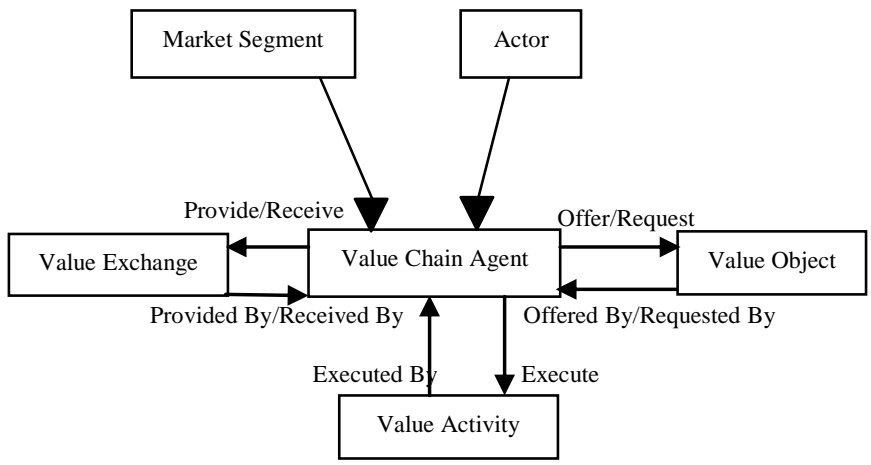

Fig 8: Concepts and their relations in $\mathrm{e}^{3}$-value in global and activity view 


\section{Evaluation}

According to some metrics in [21], mapping between mentioned ontologies, based on omen and extended omen is evaluated with previous works $[5,6]$ the result is in table 1 .

Table 1.Mapping based on Extended Omen and Omen in compare with previous works

\begin{tabular}{|l|l|l|l|l|}
\hline & {$[5]$} & {$[6]$} & omen & $\begin{array}{l}\text { Extended } \\
\text { omen }\end{array}$ \\
\hline stable & - & - & $\checkmark$ & $\checkmark$ \\
\hline intelligent & $\sqrt{ }$ & $\sqrt{ }$ & $\sqrt{ }$ & $\checkmark$ \\
\hline Discriminating & $\sqrt{ }$ & $\times$ & $\times$ & $\times$ \\
\hline
\end{tabular}

Because threshold is just defined for mapping framework so for references [5, 6], cannot declare stability. Omen and extended omen have stability because; threshold is just defined once for them. All of them are intelligent because they do not map based on string character similarity. Except reference [5], none of them are discriminating and one concept in one ontology could be mapped to more than one concept in another ontology.

\section{Case Study}

For case study, a company which produces a product and provides it's equipments from its partner is considered.

According to the case study, concepts in REA are:

Resource $=$ money, equipment, product, human resource

Agent $=$ customer, company, partner

Event $=$ produce of the products, send money, receive money, send equipment, receive equipment, send product, receive product

According to the case study, concepts in BMO are:

Value Configuration= produce of the products, send money, receive money, send equipment, receive equipment, send product

Value Proposition $=$ product

Actor $=$ partner, company

According to the case study, concepts in $\mathrm{e}^{3}$-value are:

Value Object $=$ product, money, equipment

Value Exchange $=$ send money, receive money, send equipment, receive equipment, send product, receive product

Actor $=$ partner, company

Market Segment $=$ customer

Value Activity= produce of the products

With limitation events in REA to events which are executed by the company or partner its concepts would be:

Resource $=$ money, equipment, product, human resource Agent $=$ customer, company, partner

Event $=$ produce of the products, send money, receive money, send equipment, receive equipment, send product

So Event in REA is mapped to Value Configuration in BMO, Actor in BMO is a subclass of Agent in REA and Value Proposition in BMO is a subclass of Resource in REA.

If just consider transfer events in REA, its concepts are:

Resource $=$ money, equipment, product

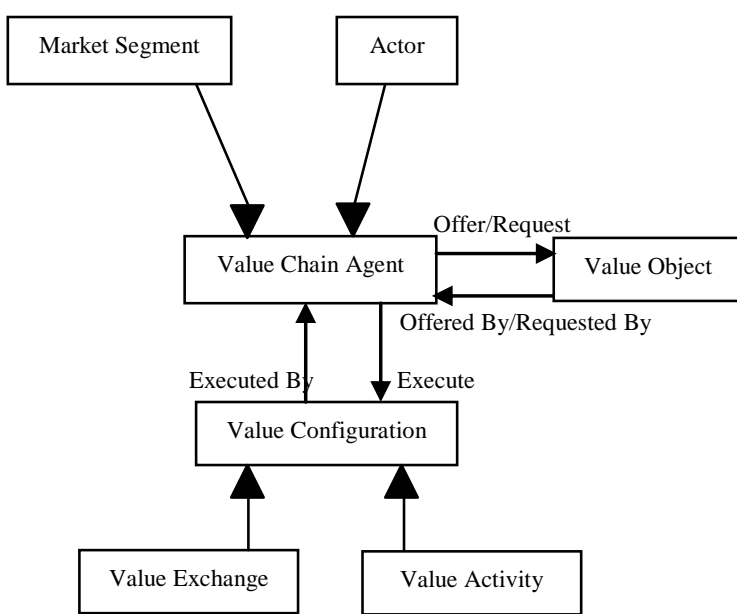

Fig 9: Concepts and their relations in $\mathrm{e}^{3}$-value in global and activity view with Value Configuration

Agent $=$ customer, company, partner

Event $=$ send money, receive money, send equipment, receive equipment, send product

So Event in REA is mapped to Value Exchange in $\mathrm{e}^{3}$-value, Actor and Market Segment in $\mathrm{e}^{3}$-value are subclasses of Agent in REA and Value Object in $\mathrm{e}^{3}$-value is mapped to Resource in REA.

With limitation of value chain agent in $\mathrm{e}^{3}$-value to the company or the partner, its concepts would be:

Value Object= product, money, equipment

Value Exchange $=$ send money, receive money, send equipment, receive equipment, send product

Actor $=$ partner, company

Value Activity $=$ produce of the products

So Actor in $\mathrm{e}^{3}$-value is mapped to Actor in BMO, Value Exchange and Value activity in $\mathrm{e}^{3}$-value are subclasses of Value Configuration in BMO.

According to the case study, can conclude the correction of the proposing mapping based on extended omen.

\section{Conclusion and future works}

Interoperability in cross enterprise collaboration is so important. Cross enterprise collaboration consists of different business domains which use different ontologies; there are three main business domain ontologies (REA, $\mathrm{e}^{3}$-value and BMO). Different business domains that use different ontologies in a cross enterprise collaboration cannot be
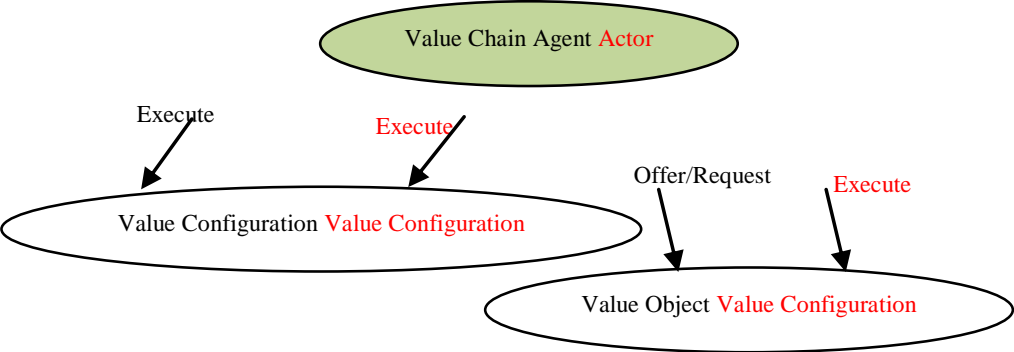

Fig 10: Nodes and their relations in mapping between BMO and $\mathrm{e}^{3}$-value 
Interoperable to each other because they do not have common concepts so proposing a mapping between these ontologies is essential. The proposed mapping must be based on framework for reliability. Omen was selected as a mapping framework for considering association relationships. In this paper an additional Meta rule is added to omen for reaching better mapping between mentioned ontologies and proposed a new framework which is called extended omen.

Extended omen likes omen needs evidence nodes for enhancing mapping between ontologies, so in this paper, first a pair of mapping between concepts of two ontologies were defined, then with meta rules in extended omen the mapping are enhanced.

For future work, suggest the followings:

- Omen is just used for mapping between mentioned ontologies and it can be used for mapping between other ontologies, even in other domains.

- $\quad$ As mapping frameworks in semantic web are growing fast, so can try other frameworks for mapping between ontologies.

- Add more Meta rules to omen for receiving much correct mappings.

- More concepts like duality, commitment and contract could be considered in REA for mapping.

\section{References}

[1] J. Gordjin, "Value based requirements engineering exploring innovative e_comerce ideas," PhD Thesis, University of Vrije, Amsterdam, Netherlands, August 2002.

[2] T. Ilayperuma, "Reference ontology for business models," PhD Thesis, University of Lausanne, Lausanne, Switzerland, May 2004.

[3] A. Osterwalder, "The business model ontology," $\mathrm{PhD}$ Thesis, University of Stockholm, Stockholm, Sweden, 2007.

[4] W.E. McCarthy, "The REA accounting model: a generalized framework for accounting systems in a shared data environment," The Accounting Review, Vol. LVII, No. 3, July 1982.S. Purser, "A practical guide to managing information security", Artech House Technology Management Library, 2004.

[5] R. Schuster, T. Motal, "From e $\mathrm{e}^{3}$-value to REA: modeling multi-party ebusiness collaborations," IEEE Conference on Commerce and Enterprise Computing, pp. 202-208, 2009.

[6] J. Gordijin, A. Osterwalder, Y. Pigneur, "Comparing two business model ontologies for designing e-business and value constellations," 18th Bled eConferenceeIntegration in Action, Bled, Slovenia, 2005.

[7] P. Mitra, G. Wiederhold , J. Jannink, "Semi-automatic integration of knowledge sources, " 2nd International Conference on Information Fusion, pp. 572-581, Sunnyvale , 1999.
[8] P. Mitra, G. Wiederhold , M. Kersten, "A graph oriented model for articulation of ontology interdependencies," 8th Conference on Extending Database Technology, vol. 1777, pp. 86-100, Praha, 2000.

[9] P. Mitra, N. Noy , A. Jaiswal, “Ontology mapping discovery with uncertainty, "4th International Semantic Web Conference, vol. 3729, pp. 537-547, Galway, 2005.

[10] J. Euzenat, "Brief overview of $t$-tree: the tropes taxonomy building tool," 4th ASIS SIG/CR Workshop on Classification Research, Columbus, pp. 69-87, 1994.

[11] A. Doan, J. Madhavan, P. Domingos , A. Halevy, "ontology matching: a machine learning approach," 8th International Semantic Web Conference, pp. 385-404, Springer, Verlag, Berlin, 2004.

[12] J. Euzenat , P. Valtchev, "Similarity-based ontology alignment in OWL-lite, " 15th European Conference on Artificial Intelligence, pp. 333-337, Valencia, 2004.

[13] Y. Li, Q. Zhong , J. Tang, "Results of ontology alignment with RiMOM, " International workshop on Ontology Matching, Busan, Korea, pp. 227-235. November 2007.

[14] P. Bouquet, L. Serafini, S. Zanobini , S. Sceffer, "Bootstrapping semantics on the web: meaning elicitation from schemas, "15th International WWW Conference, pp. 505-512, Edinburgh (UK), 2006.

[15] M. Lacher , G. Groh, "Facilitating the exchange of explicit knowledge through ontology mappings, " 14th International Florida Artificial Intelligence Research Society Conference (FLAIRS), pp. 305-309, Key West (FL US), 2001.

[16] T. Bach, R. Kuntz , F. Gandon, "On ontology matching problems (for building a corporate semantic web in a multi-communities organization), "6th International Conference on Enterprise Information Systems (ICEIS), pp. 236-243, Porto (PT), 2004.

[17] F. Giunchiglia , P. Shvaiko, "Semantic matching," The Knowledge Engineering Review, pp. 265-280, 2003.

[18] Y. Kalfoglou , M. Schorlemmer, "IF-Map: an ontology mapping method based on information flow theory," Journal on Data Semantics, 2003.

[19] U. Straccia , R. Troncy, "oMAP: Combining classifiers for aligning automatically OWL ontologies, "6th International Conference on Web Information Systems Engineering (WISE), pp. 133-147, New York (NY US) 2005.

[20] G. Frederik, L. Wim , P. Geert, "Positioning REA as a business domain ontology, " 25th Conference on Resource Event Agent, Newark, Delaware, USA,2007.

[21] S. Giorgos and K. Stefanos, "A String Metric for Ontology Alignment," 10th international semantic web conference, pp. 624-637, Springer ,Berlin, Heidelberg, 2005. 